\title{
EXPLICIT BARYCENTRIC WEIGHTS FOR POLYNOMIAL INTERPOLATION IN THE ROOTS OR EXTREMA OF CLASSICAL ORTHOGONAL POLYNOMIALS
}

\author{
HAIYONG WANG, DAAN HUYBRECHS, AND STEFAN VANDEWALLE
}

\begin{abstract}
Barycentric interpolation is arguably the method of choice for numerical polynomial interpolation. The polynomial interpolant is expressed in terms of function values using the so-called barycentric weights, which depend on the interpolation points. Few explicit formulae for these barycentric weights are known. In [H. Wang and S. Xiang, Math. Comp., 81 (2012), 861-877], the authors have shown that the barycentric weights of the roots of Legendre polynomials can be expressed explicitly in terms of the weights of the corresponding Gaussian quadrature rule. This idea was subsequently implemented in the Chebfun package [L. N. Trefethen and others, The Chebfun Development Team, 2011] and in the process generalized by the Chebfun authors to the roots of Jacobi, Laguerre and Hermite polynomials. In this paper, we explore the generality of the link between barycentric weights and Gaussian quadrature and show that such relationships are related to the existence of lowering operators for orthogonal polynomials. We supply an exhaustive list of cases, in which all known formulae are recovered and also some new formulae are derived, including the barycentric weights for Gauss-Radau and Gauss-Lobatto points. Based on a fast $\mathcal{O}(n)$ algorithm for the computation of Gaussian quadrature, due to Hale and Townsend, this leads to an $\mathcal{O}(n)$ computational scheme for barycentric weights.
\end{abstract}

\section{INTRODUCTION}

Polynomial interpolation is a fundamental tool in many areas of numerical analysis [4, 6, 9, 24, 27. It is usually introduced using the Lagrange form of the interpolating polynomial, as follows. Let $\left\{x_{j}\right\}_{j=0}^{n}$ be a set of distinct nodes. Then the polynomial of degree $n$ that interpolates the function $f(x)$ at these points may be written as

$$
p_{n}(x)=\sum_{j=0}^{n} f\left(x_{j}\right) \ell_{j}(x)
$$

where

$$
\ell_{j}(x)=\prod_{k \neq j} \frac{x-x_{k}}{x_{j}-x_{k}}, \quad j=0,1, \ldots, n,
$$

are the Lagrange fundamental polynomials. The Lagrange form of the interpolating polynomial (1.1) is not advocated for numerical computations, as typical algorithms require $\mathcal{O}\left(n^{2}\right)$ operations. Moreover, they are numerically unstable and each time a node $x_{j}$ is modified or added, all Lagrange fundamental polynomials have to be recalculated [1].

Received by the editor November 12, 2012 and, in revised form, March 22, 2013.

2010 Mathematics Subject Classification. Primary 41A05, 65D05, 65D15. 
In order to obtain good approximations via interpolation, the choice of interpolation nodes is particularly important. For example, it is well-known that equispaced points give rise to the Runge phenomenon when the number of interpolation points is large. The interpolating polynomial diverges, even when the function $f$ to be interpolated is analytic. In order to avoid the occurrence of Runge's phenomenon, various techniques have been proposed over the past decades and we refer the reader to [20] for a comprehensive discussion. In practice, the interpolation nodes with the density distribution $1 / \sqrt{1-x^{2}}$ are optimal in various senses for polynomial approximation on $[-1,1]$ and they typically lead to well-behaved Lagrange interpolation [27. Good candidates are the roots and extrema of certain orthogonal polynomials.

Interpolation approximations based on the roots of orthogonal polynomials are widely applied in numerical integration, spectral methods, etc. For computational purposes, an alternative and more preferable approach to the Lagrange form is to rewrite the interpolation polynomial as a sum of the corresponding orthogonal polynomials (see, for example, 7, 18,22]). Let $\left\{\pi_{n}(x)\right\}_{n \in \mathbb{N}}$ be a sequence of polynomials orthogonal with respect to a given nonnegative and integrable weight function $\omega(x)$ on $(a, b)$, and

$$
\int_{a}^{b} \omega(x) \pi_{m}(x) \pi_{n}(x) d x=h_{n} \delta_{m n}, \quad m, n \geq 0,
$$

where $h_{n}$ is a positive normalization constant and $\delta_{m n}$ is the Kronecker delta. Suppose that the interpolation nodes $\left\{x_{j}\right\}_{j=0}^{n}$ are the zeros of $\pi_{n+1}(x)$, then the interpolating polynomial $p_{n}(x)$ can be written as a linear combination of $\left\{\pi_{k}(x)\right\}_{k=0}^{n}$,

$$
p_{n}(x)=\sum_{k=0}^{n} a_{k} \pi_{k}(x)
$$

where the coefficients $a_{k}$ are given by

$$
a_{k}=h_{k}^{-1} \sum_{j=0}^{n} w_{j} f\left(x_{j}\right) \pi_{k}\left(x_{j}\right), \quad k=0, \ldots, n,
$$

and $\left\{w_{j}\right\}_{j=0}^{n}$ are the Gaussian quadrature weights corresponding to the weight $\omega(x)$. Although this form of the interpolating polynomial is numerically stable, the calculation of the coefficients $\left\{a_{k}\right\}_{k=0}^{n}$ takes $\mathcal{O}\left(n^{2}\right)$ operations by direct evaluation. In the special case of Chebyshev points, the cost can be reduced to $\mathcal{O}(n \log n)$ using the FFT. Except for that case, using the form (1.3) leads to $\mathcal{O}\left(n^{2}\right)$ methods which makes it inefficient for large $n$.

The fast computation of the Lagrange interpolation polynomial has received substantial attention over the past decades (see [1,8, 21, 29, 31] and the references therein). From a computational point of view, it is recommended to apply the barycentric representation of the interpolating polynomial [1]. The barycentric formula, which we shall review in Section 2, equation (2.2), has several attractive features such as stability and high efficiency. For example, when the interpolation nodes are Chebyshev points of the first or second kind, the evaluation of the interpolating polynomial requires only $\mathcal{O}(n)$ operations [16, 21. For other sets of interpolation points, no explicit formulae for the barycentric weights are known. Direct computation of the barycentric weights again requires $\mathcal{O}\left(n^{2}\right)$ operations. 
In this paper we shall devote our attention to the study of the barycentric weights for roots and extrema of the classical orthogonal polynomials. This study is motivated by the earlier observation by the first author in [29] that a simple relationship exists between Gauss-Legendre quadrature weights $w_{j}$ and barycentric interpolation weights $\lambda_{j}^{\mathrm{Leg}}$ :

$$
\lambda_{j}^{\mathrm{Leg}}=(-1)^{j} \sqrt{\left(1-x_{j}^{2}\right) w_{j}}, \quad j=0, \ldots, n,
$$

for the case of interpolation in the roots $x_{j}$ of the Legendre polynomial of degree $n+1$. Formula (1.4) was implemented in the Chebfun package [28] as part of the legpts routine 15. Several useful generalizations were identified by the Chebfun authors in the process. In particular, formula (1.4) remains valid for the more general family of Jacobi polynomials. Furthermore, similar identities hold for the roots of Laguerre polynomials,

$$
\lambda_{j}^{\mathrm{Lag}}=(-1)^{j} \sqrt{x_{j} w_{j}}, \quad j=0, \ldots, n,
$$

and Hermite polynomials,

$$
\lambda_{j}^{\mathrm{H}}=(-1)^{j} \sqrt{w_{j}}, \quad j=0, \ldots, n,
$$

see routines legpts, chebpts, jacpts, lagpts and hermpts in Chebfun. These developments are detailed in [15] and [27, pp. 151-152].

The motivation for formula (1.4) was based on an explicit form of the GaussLegendre quadrature weights; see [29, Thm. 3.1]. The above formulae lead to the following question: How general is the link between barycentric weights and weights of Gaussian quadrature? For this purpose, we describe an alternative analysis leading to (1.4) (1.6) using the notion of lowering operators of orthogonal polynomials. This yields additional formulae for interpolation at the extremae of the classical orthogonal polynomials (Jacobi, Laguerre and Hermite), corresponding to the Radau and Lobatto variants of Gaussian quadrature.

Hence, the computation of the barycentric weights has been transformed into the computation of the nodes and weights of Gaussian quadrature rules, for which we can apply the well-known Golub-Welsch algorithm [13] in $\mathcal{O}\left(n^{2}\right)$ operations. Faster algorithms have been described with optimal $\mathcal{O}(n)$ computational complexity for specific cases. The first such algorithm is due to Glaser, Liu and Rokhlin, requiring only $\mathcal{O}(n)$ operations for the computation of Gaussian quadrature for the classical polynomials 12. More recently, two approaches have been described with the same optimal complexity, but with much reduced constants, based on exploiting known asymptotic behaviour of orthogonal polynomials. The approach of Hale and Townsend includes the case of Gauss-Jacobi quadrature [14. Bogaert, Michiels and Fostier describe a similar approach for Gauss-Legendre quadrature [2]. As a result of these developments, it is evident that the polynomial interpolants in the roots or extrema of Jacobi polynomials can be computed in only $\mathcal{O}(n)$ operations as well by using their barycentric representations.

This paper is organized as follows. In the next section, we start with some known results about the barycentric interpolation formula and present a derivation of the barycentric weights for the zeros of orthogonal polynomials. In Section 3 , we explore the explicit forms of the barycentric weights for the zeros of orthogonal polynomials with some additional points. We give several numerical examples in Section 4 and conclude with some remarks in Section 5. 


\section{BARYCENTRIC INTERPOLATION FORMULA}

2.1. First and second barycentric interpolation formula. In this section we review some facts about the barycentric interpolation formula. Let

$$
\ell(x)=\left(x-x_{0}\right)\left(x-x_{1}\right) \cdots\left(x-x_{n}\right)
$$

be the monic polynomial of degree $n+1$ that vanishes at the interpolation nodes $x_{j}$. Then the Lagrange form of the interpolating polynomial $p_{n}(x)$ can be rewritten in barycentric form as

$$
p_{n}(x)=\frac{\sum_{j=0}^{n} \frac{\lambda_{j}}{x-x_{j}} f\left(x_{j}\right)}{\sum_{j=0}^{n} \frac{\lambda_{j}}{x-x_{j}}},
$$

where the barycentric weights are defined by [23, p. 218]

$$
\lambda_{j}=\frac{1}{\Pi_{j \neq k}\left(x_{j}-x_{k}\right)}=\frac{1}{\ell^{\prime}\left(x_{j}\right)}, \quad j=0,1, \ldots, n .
$$

Expression (2.2) is the so-called second form of the barycentric formula. Due to the division, the barycentric weights can be simplified by cancelling the common factors without altering the result. We will call the resulting weights the simplified barycentric weights.

The first form of the barycentric formula is given by

$$
p_{n}(x)=\ell(x) \sum_{j=0}^{n} \frac{\lambda_{j}}{x-x_{j}} f\left(x_{j}\right),
$$

with the weights still defined by (2.3). A disadvantage in this case is that common factors of $\lambda_{j}$ may not be cancelled, which leads to more complicated expressions later on. On the other hand, it is shown recently in [30] that the second formula is not stable for (complex) values of $x$ away from the interpolation interval, whereas the first formula is. For this reason we include results for the full barycentric weights defined by (2.3) as well as the simplified ones.

For convenience, we assume throughout this paper that the interpolation nodes $x_{j}$ are monotonic and hence, the barycentric weights $\lambda_{j}$ always have alternating signs. For points inside the interpolation interval, the barycentric formula (2.2) has been proved to be numerically stable for any set of interpolating points with a small Lebesgue constant [17]. For a general set of interpolation nodes, the computation of the barycentric weights $\left\{\lambda_{j}\right\}_{j=0}^{n}$ requires $\mathcal{O}\left(n^{2}\right)$ operations 31. However, for several important sets of points such as Chebyshev points of the first and second kind, explicit formulae for these barycentric weights $\lambda_{j}$ are known. For example, for the Chebyshev points of the first kind

$$
x_{j}=\cos \left(\frac{2 j+1}{2 n+2} \pi\right), \quad j=0,1, \ldots, n,
$$

the simplified barycentric weights are given by [16, p. 249]

$$
\lambda_{j}^{\mathrm{CH} 1}=(-1)^{j} \sin \left(\frac{2 j+1}{2 n+2} \pi\right) .
$$


For the Chebyshev points of the second kind

$$
x_{j}=\cos \left(\frac{j}{n} \pi\right), \quad j=0,1, \ldots, n,
$$

the simplified barycentric weights are given by 21$]$

$$
\lambda_{j}^{\mathrm{CH} 2}=(-1)^{j} \delta_{j}, \quad \delta_{j}=\left\{\begin{array}{cc}
1 / 2, & j=0 \text { or } j=n, \\
1, & \text { otherwise. }
\end{array}\right.
$$

Thus, each evaluation of the interpolation formulae $p_{n}(x)$ for Chebyshev points can be implemented in only $\mathcal{O}(n)$ operations.

2.2. Explicit barycentric weights in terms of Gaussian quadrature. Equation (2.3) shows that the barycentric weights are given in terms of the derivative of the polynomial $\ell(x)$ that vanishes at the interpolation nodes. In the following we consider the case where $\ell(x)$ is an orthogonal polynomial with respect to a weight function $w(x)$.

Let $\left\{x_{j}\right\}_{j=0}^{n}$ be the $n+1$ roots of the polynomial $\pi_{n+1}(x)$ and $k_{n}$ be the leading coefficient of $\pi_{n}(x)$. The corresponding Gaussian quadrature rule is

$$
\int_{a}^{b} \omega(x) f(x) d x \simeq \sum_{j=0}^{n} w_{j} f\left(x_{j}\right),
$$

where the Gaussian quadrature weights are given by [7, p. 97]

$$
w_{j}=\frac{k_{n+1} h_{n}}{k_{n} \pi_{n+1}^{\prime}\left(x_{j}\right) \pi_{n}\left(x_{j}\right)},
$$

with $h_{n}$ defined as in (1.2). When the roots $x_{j}$ are used for interpolation purposes, the corresponding barycentric weights can be written in the following form:

$$
\lambda_{j}=\frac{k_{n+1}}{\pi_{n+1}^{\prime}\left(x_{j}\right)} .
$$

Combining this with (2.8) leads to

$$
\lambda_{j}=\frac{k_{n}}{h_{n}} \pi_{n}\left(x_{j}\right) w_{j} .
$$

This relation between barycentric weights and Gaussian weights does not immediately lead to faster computations, as one still has to evaluate the orthogonal polynomial $\pi_{n}$ in all the nodes $x_{j}$. Based, for example, on recurrence relations for the polynomials, this step still requires $\mathcal{O}\left(n^{2}\right)$ operations.

The basic observation underlying the remainder of this paper is that for the classical polynomials $\pi_{n}\left(x_{j}\right)$ can be written in terms of $\pi_{n+1}^{\prime}\left(x_{j}\right)$. Equation (2.9) can be used again to remove the $\pi_{n}\left(x_{j}\right)$ factor from (2.10), and as a result the barycentric weights are only related to the nodes and weights of corresponding Gaussian quadrature rule.

We prepare the setting and establish notation with the following lemma.

Lemma 2.1. Let $\pi_{n}(x)$ satisfy the equation of hypergeometric type

$$
\varphi(x) \pi_{n}{ }^{\prime \prime}(x)+\phi(x) \pi_{n}{ }^{\prime}(x)+\nu_{n} \pi_{n}(x)=0,
$$

where $\varphi(x)$ and $\phi(x)$ are polynomials of at most second and first degree respectively. When $\nu_{n}=-n \phi^{\prime}(x)-\frac{n(n-1)}{2} \varphi^{\prime \prime}(x)$, the above equation has a particular solution 
$\pi_{n}(x)$ which is a polynomial of degree $n$, and all orders of the derivatives of $\pi_{n}(x)$ have the following Rodrigues formula,

$$
\pi_{n}^{(m)}(x)=\frac{A_{m n} B_{n}}{\varphi^{m}(x) \omega(x)} \frac{d^{n-m}}{d x^{n-m}}\left[\varphi^{n}(x) \omega(x)\right],
$$

where

$$
A_{m n}=\frac{n !}{(n-m) !} \prod_{k=0}^{m-1}\left(\phi^{\prime}(x)+\frac{1}{2}(n+k-1) \varphi^{\prime \prime}(x)\right), \quad A_{0 n}=1,
$$

and $B_{n}$ is a normalizing constant

$$
B_{n}=k_{n} \prod_{k=0}^{n-1}\left(\phi^{\prime}(x)+\frac{1}{2}(n+k-1) \varphi^{\prime \prime}(x)\right)^{-1}, \quad B_{0}=k_{0} .
$$

The function $\omega(x)$ is chosen such that

$$
(\varphi(x) \omega(x))^{\prime}=\phi(x) \omega(x) .
$$

Moreover, $\pi_{n}(x)$ is orthogonal with respect to the function $\omega(x)$.

Proof. See [19, p. 24].

Note that the polynomials satisfying an equation of the form (2.11) with the right conditions are precisely the classical orthogonal polynomials: Jacobi polynomials (which include Legendre, Chebyshev and Gegenbauer polynomials), Laguerre polynomials and Hermite polynomials. The following theorem gives the general relation between barycentric weights and Gaussian quadrature.

Theorem 2.2. Let $\pi_{n}(x)$ satisfy the above conditions. Then the barycentric weights $\lambda_{j}$ corresponding to the roots of $\pi_{n+1}(x)$ are given by

$$
\lambda_{j}=\sigma(-1)^{j} \sqrt{\frac{k_{n+1}^{2}(2 n+2) \varphi\left(x_{j}\right) w_{j}}{\nu_{2 n+2} h_{n+1}}}, \quad j=0,1, \ldots, n,
$$

where $\sigma=+1$ for even $n$ and $\sigma=-1$ for odd $n$.

Proof. Set $\phi_{n}(x)=\phi(x)+n \varphi^{\prime}(x)$. It then follows from (2.14) that

$$
\left(\varphi(x)^{n+1} \omega(x)\right)^{\prime}=\phi_{n}(x) \varphi(x)^{n} \omega(x) .
$$

Applying the Rodrigues formula (2.12) and noting that $\phi_{n}(x)$ is a polynomial of degree one, we have

$$
\begin{aligned}
\pi_{n+1}(x) & =\frac{B_{n+1}}{\omega(x)} \frac{d^{n+1}}{d x^{n+1}}\left[\varphi^{n+1}(x) \omega(x)\right] \\
& =\frac{B_{n+1}}{\omega(x)} \frac{d^{n}}{d x^{n}}\left[\phi_{n}(x) \varphi(x)^{n} \omega(x)\right] \\
& =\frac{B_{n+1}}{\omega(x)}\left[\phi_{n}(x)\left(\varphi(x)^{n} \omega(x)\right)^{(n)}+n \phi_{n}{ }^{\prime}(x)\left(\varphi(x)^{n} \omega(x)\right)^{(n-1)}\right] \\
& =\frac{B_{n+1}}{B_{n}}\left[\phi_{n}(x) \pi_{n}(x)+\frac{n \phi_{n}{ }^{\prime}(x)}{A_{1 n}} \varphi(x) \pi_{n}{ }^{\prime}(x)\right] .
\end{aligned}
$$


Consequently,

$$
\varphi(x) \pi_{n}^{\prime}(x)=\frac{A_{1 n}}{n \phi_{n}{ }^{\prime}(x)}\left[\frac{B_{n}}{B_{n+1}} \pi_{n+1}(x)-\phi_{n}(x) \pi_{n}(x)\right] .
$$

Recall that $\pi_{n}(x)$ is orthogonal with respect to the function $\omega(x)$. It has the threeterm recurrence relation

$$
x \pi_{n}(x)=\alpha_{n} \pi_{n+1}(x)+\beta_{n} \pi_{n}(x)+\gamma_{n} \pi_{n-1}(x),
$$

where $\alpha_{n}, \beta_{n}$ and $\gamma_{n}$ are constants. Using the orthogonality of $\pi_{n}(x)$, we can easily find that

$$
\alpha_{n}=\frac{k_{n}}{k_{n+1}}, \quad \gamma_{n}=\frac{h_{n} k_{n-1}}{k_{n} h_{n-1}} .
$$

Combining (2.17) and (2.18) yields

$$
\begin{aligned}
\varphi(x) \pi_{n}^{\prime}(x) & =\frac{A_{1 n}}{n \phi_{n}^{\prime}(x)}\left[\frac{B_{n}}{B_{n+1} \alpha_{n}}\left(x-\beta_{n}\right)-\phi_{n}(x)\right] \pi_{n}(x) \\
& -\frac{A_{1 n} B_{n} \gamma_{n}}{n \phi_{n}^{\prime}(x) B_{n+1} \alpha_{n}} \pi_{n-1}(x) .
\end{aligned}
$$

Furthermore, taking the definition (2.13) into account and using the fact that

$$
A_{1 n}=n\left(\phi^{\prime}(x)+\frac{n-1}{2} \varphi^{\prime \prime}\right)=-\nu_{n}, \quad \phi_{n}^{\prime}(x)=\phi^{\prime}(x)+n \varphi^{\prime \prime}(x)=-\frac{\nu_{2 n+1}}{2 n+1},
$$

we obtain

$$
\begin{aligned}
\varphi(x) \pi_{n}{ }^{\prime}(x) & =-\frac{(2 n+1) \nu_{n}}{n \nu_{2 n+1}}\left[\frac{\nu_{2 n} \nu_{2 n+1}}{2 \nu_{n}(2 n+1)}\left(x-\beta_{n}\right)+\phi_{n}(x)\right] \pi_{n}(x) \\
& +\frac{\nu_{2 n} h_{n} k_{n-1}}{2 n k_{n} h_{n-1}} \pi_{n-1}(x) .
\end{aligned}
$$

Let $x_{j}, j=0, \ldots, n$, be the roots of $\pi_{n+1}(x)$. Replacing $n$ with $n+1$ in (2.21), it follows that

$$
\varphi\left(x_{j}\right) \pi_{n+1}^{\prime}\left(x_{j}\right)=\frac{\nu_{2 n+2} k_{n} h_{n+1}}{(2 n+2) k_{n+1} h_{n}} \pi_{n}\left(x_{j}\right) .
$$

This, together with (2.9) and (2.10), implies that

$$
\lambda_{j}^{2}=\frac{k_{n+1}^{2}(2 n+2) \varphi\left(x_{j}\right) w_{j}}{\nu_{2 n+2} h_{n+1}} .
$$

Recalling that the barycentric weights $\lambda_{j}$ have alternating signs, expression (2.15) follows.

We make some further comments regarding Theorem 2.2 and its proof, in order to put the result itself, as well as its scope and limitations, in a proper context:

- The crucial identity that relates $\pi_{n}^{\prime}$ to $\pi_{n}$ and $\pi_{n-1}$ is given by expression (2.21). This is an example of a so-called lowering operator. Indeed, moving $\pi_{n}$ to the left-hand side in (2.21) defines an operator that acts on $\pi_{n}$ and that yields a polynomial of lower degree - hence the name. We have included a typical derivation of the lowering operator in the proof for the purpose of being self-contained. A classical reference is [19]. 
- The derivatives of classical polynomials $\pi_{n}^{(k)}$ are again orthogonal on the same interval with respect to the new weight function $\varphi(x)^{k} \omega(x)$ [19]. Thus if the interpolation points are roots of $\pi_{n}^{(k)}, n>k$, then lowering operators can also be used to write the barycentric weights in terms of the nodes and weights of the Gaussian quadrature with respect to the new weight function.

- More general lowering operators can be found for other kinds of orthogonal polynomials as well, leading to identities similar to (2.21). Examples include polynomials orthogonal with respect to the weight function $e^{-V(x)}$ on $[-1,1]$, where $V(x)$ is a polynomial $[3$, Eq. (1.5)], and polynomials orthogonal with respect to the weight function $w(x) e^{-V(x)}$, where $w(x)$ is any of the classical weight functions [26, Eq. (6.5)]. Any such identity immediately gives rise to a relationship between barycentric weights and Gaussian quadrature weights.

- However, it is important to point out that the lowering operator in general depends on $n$. This dependence is benign in the setting of Theorem 2.2, in the sense that all $n$-dependent quantities still appearing in the final result (2.15) have explicit expressions (which we supply further on in $\$ 2.3$ ). In particular, these quantities can be evaluated in a number of operations that do not depend on $n$. Unfortunately, this is no longer the case for the lowering operators in 3,26 .

- Furthermore, the algorithms for the fast construction of Gaussian quadrature rules are only applicable for the classical orthogonal polynomials [2, 12, 14. Thus, an $\mathcal{O}(n)$ algorithm for the computation of barycentric weights from the Gaussian weights cannot be immediately generalized to other polynomials.

The last two comments are the two reasons for restricting ourselves to the classical orthogonal polynomials in Lemma 2.1 and Theorem 2.2 above.

\subsection{Explicit barycentric weights for the classical orthogonal polynomials.}

As mentioned above, polynomials satisfying an equation of the form (2.11) are the classical polynomials. For Jacobi polynomials, we have [25, p. 61]

$\left(1-x^{2}\right) P_{n}^{(\alpha, \beta) \prime \prime}(x)+[\beta-\alpha-(\alpha+\beta+2) x] P_{n}^{(\alpha, \beta) \prime}(x)+n(n+\alpha+\beta+1) P_{n}^{(\alpha, \beta)}(x)=0$,

from which we obtain

$$
\varphi(x)=1-x^{2}, \quad \nu_{n}=n(n+\alpha+\beta+1) .
$$

Substituting these into (2.15) and recalling that

$k_{n+1}=\frac{1}{2^{n+1}}\left(\begin{array}{c}2 n+\alpha+\beta+2 \\ n+1\end{array}\right), \quad h_{n+1}=\frac{2^{\alpha+\beta+1}}{2 n+\alpha+\beta+3} \frac{\Gamma(n+\alpha+2) \Gamma(n+\beta+2)}{(n+1) ! \Gamma(n+\alpha+\beta+2)}$,

we obtain the barycentric weights. Cancelling the common factors yields the simplified weights. In the next three corollaries, $\sigma$ is defined as in Theorem 2.2

Corollary 2.3. The barycentric weights for the roots of the Jacobi polynomial $P_{n+1}^{(\alpha, \beta)}(x)$ are

$$
\lambda_{j}^{(\alpha, \beta)}=C_{n}^{(\alpha, \beta)}(-1)^{j} \sqrt{\left(1-x_{j}^{2}\right) w_{j}}, \quad j=0, \ldots, n,
$$


where $C_{n}^{(\alpha, \beta)}=1$ for the simplified weights, and otherwise

$$
\begin{aligned}
C_{n}^{(\alpha, \beta)} & =\sigma \frac{\Gamma(2 n+\alpha+\beta+3)}{2^{n+1+\frac{\alpha+\beta+1}{2}}} \\
& \times \frac{1}{\sqrt{(n+1) ! \Gamma(n+\alpha+\beta+2) \Gamma(n+\alpha+2) \Gamma(n+\beta+2)}} .
\end{aligned}
$$

The case of Legendre polynomials corresponds to the choice $\alpha=\beta=0$. Formula (2.24) indeed corresponds to expression (1.4), which was observed earlier in [29], for the case of the simplified weights. Note that the formula for the simplified weights remains unchanged for the general Jacobi case.

Similarly, the Laguerre polynomials satisfy the following differential equations [25, p. 100]:

and

$$
x L_{n}^{(\alpha) \prime \prime}(x)+(1+\alpha-x) L_{n}^{(\alpha) \prime}(x)+n L_{n}^{(\alpha)}(x)=0
$$

$$
k_{n+1}=\frac{(-1)^{n+1}}{(n+1) !}, \quad h_{n+1}=\frac{\Gamma(n+\alpha+2)}{(n+1) !} .
$$

These lead to the following expressions.

Corollary 2.4. The barycentric weights for the roots of the Laguerre polynomial $L_{n+1}^{(\alpha)}(x)$ are

$$
\lambda_{j}^{\mathrm{Lag}}=C_{n}^{(\alpha)}(-1)^{j} \sqrt{x_{j} w_{j}}, \quad j=0, \ldots, n,
$$

where $C_{n}^{(\alpha)}=1$ for the simplified weights, and otherwise

$$
C_{n}^{(\alpha)}=\sigma \frac{1}{\sqrt{\Gamma(n+\alpha+2)(n+1) !}} .
$$

Finally, the Hermite polynomials satisfy [25, p. 106]

$$
H_{n}{ }^{\prime \prime}(x)-2 x H_{n}{ }^{\prime}(x)+2 n H_{n}(x)=0,
$$

from which we deduce that

$$
\varphi(x)=1, \quad \nu_{n}=2 n .
$$

Moreover, using the fact that

$$
k_{n+1}=2^{n+1}, \quad h_{n+1}=\sqrt{\pi} 2^{n+1}(n+1) !,
$$

we obtain the following corollary.

Corollary 2.5. The barycentric weights for the roots of the Hermite polynomial $H_{n+1}(x)$ are

$$
\lambda_{j}^{\mathrm{H}}=C_{n}(-1)^{j} \sqrt{w_{j}}, \quad j=0, \ldots, n,
$$

where $C_{n}=1$ for the simplified weights, and otherwise

$$
C_{n}=\sigma \sqrt{\frac{2^{n}}{(n+1) ! \sqrt{\pi}}} .
$$

The above corollaries show a close and simple connection between the barycentric weights and the nodes and weights of the corresponding Gaussian quadrature rule for all classical orthogonal polynomials. Since the Legendre polynomials and the Chebyshev polynomials of the first and second kinds are all special cases of Jacobi polynomials, the barycentric weights for the zeros of these polynomials, given in Section 1 can be derived as immediate consequence. 


\section{Additional interpolation points}

A set of Gaussian quadrature points is sometimes augmented with a small set of additional points. Two useful examples are Gauss-Radau and Gauss-Lobatto rules, where one or two (respectively) of the endpoints of the integration interval are added to the set of quadrature points. The weights of the Radau and Lobatto variants can be written in terms of the weights of a regular Gaussian quadrature rule. We show that in some cases one can also write the barycentric weights in terms of the Gauss-Radau or Gauss-Lobatto quadrature weights. This setting covers the set of Chebyshev points of the second kind, i.e., the set of all maxima of the Chebyshev polynomials of the first kind on $[-1,1]$, for which explicit formulae for the barycentric weights are already known.

3.1. Gaussian quadrature with preassigned abscissae. We start out in a more general setting in order to illustrate the scope of the arguments. We study Gaussian quadrature rules with a number of preassigned abscissae, for example the set $\left\{y_{j}\right\}_{j=1}^{m}$. We are interested in a set of $n+1$ additional quadrature points $x_{j}$ such that the quadrature rule

$$
\int_{-1}^{1} w(x) f(x) d x \simeq \sum_{j=1}^{m} \hat{w}_{j} f\left(y_{j}\right)+\sum_{j=0}^{n} \tilde{w}_{j} f\left(x_{j}\right)
$$

is exact for polynomials up to degree $m+2 n+1$. This is known to be the maximal order [7, p. 101]. It is achieved by taking $x_{j}$ as the roots of an orthogonal polynomial $\pi_{n+1}$, if it exists, that is orthogonal in the sense that

$$
\int_{-1}^{1} w(x) r_{m}(x) \pi_{n+1}(x) x^{j} d x=0, \quad j=0, \ldots, n,
$$

where

$$
r_{m}(x)=\prod_{j=1}^{m}\left(x-y_{j}\right)
$$

is a polynomial of degree $m$ that vanishes at the preassigned quadrature nodes $y_{j}$. Note that the existence of this polynomial is not guaranteed, even for positive $w(x)$, if $r_{m}(x)$ switches sign in the integration interval.

Let $w_{j}$ be the set of weights corresponding to the regular Gaussian quadrature rule associated with $\pi_{n+1}$. The weights $\tilde{w}_{j}$ of the rule with preassigned nodes relate to $w_{j}$ in a simple way. As the following statement is not found in the standard textbook [7], we include a proof.

Lemma 3.1. We have

$$
\tilde{w}_{j}=\frac{w_{j}}{r_{m}\left(x_{j}\right)} .
$$

Proof. Since (3.1) holds for all polynomials up to degree $m+2 n+1$, we may substitute $f(x)=r_{m}(x) f_{2}(x)$, where $f_{2}(x)$ is any polynomial of degree up to $2 n+1$. The right-hand side in that case must reduce to the Gaussian rule $\sum_{j=0}^{n} w_{j} f_{2}\left(x_{j}\right)$, from which the result follows.

In the following, we will explore the corresponding generalizations of our earlier explicit expressions for the barycentric weights. Let $\lambda_{j}^{x}$ be the barycentric weights corresponding to the point set $\left\{x_{j}\right\}_{j=0}^{n}$ and $\lambda_{j}^{y}$ those corresponding to the point set $\left\{y_{j}\right\}_{j=1}^{m}$. For the combined set $\left\{x_{j}\right\}_{j=0}^{n} \cup\left\{y_{j}\right\}_{j=1}^{m}$, we denote by $\tilde{\lambda}_{j}$ the barycentric 
weights corresponding to the points $x_{j}$ and by $\hat{\lambda}_{j}$ the weights corresponding to the points $y_{j} 1$

We derive different expressions for the barycentric weights $\tilde{\lambda}_{j}$ and $\hat{\lambda}_{j}$. For the former, from (2.3) we have

$$
\tilde{\lambda}_{j}=\frac{k_{n+1}}{\pi_{n+1}^{\prime}\left(x_{j}\right) r_{m}\left(x_{j}\right)}=\frac{\lambda_{j}^{x}}{r_{m}\left(x_{j}\right)}, \quad j=0, \ldots, n,
$$

where $k_{n+1}$ is the leading order coefficient of $\pi_{n+1}(x)$. For the latter, we find

$$
\hat{\lambda}_{j}=\frac{k_{n+1}}{\pi_{n+1}\left(y_{j}\right) r_{m}^{\prime}\left(y_{j}\right)}=\lambda_{j}^{y} \frac{k_{n+1}}{\pi_{n+1}\left(y_{j}\right)}, \quad j=1, \ldots, m .
$$

Assuming $m$ is fixed and $m \ll n$, the latter case presents no computational difficulties. The orthogonal polynomial $\pi_{n+1}$ can be evaluated in $\mathcal{O}(n)$ operations at a single point, for example based on the three-term recurrence relation. Thus, evaluating $\pi_{n+1}\left(y_{j}\right)$ at the $m$ points $y_{j}$ requires only $\mathcal{O}(m n)$ operations. The barycentric weights $\lambda_{j}^{y}$ are easily computed in at most $\mathcal{O}\left(m^{2}\right)$ operations. Hence, we focus on the weights given by (3.3). We will consider a number of interesting cases in which roots of classical orthogonal polynomials are supplemented with additional interpolation points.

3.2. Gauss-Lobatto variant for Jacobi polynomials. Let us consider first the Gauss-Lobatto points associated with Jacobi polynomials. Thus, we consider the additional points $\left\{y_{j}\right\}=\{-1,1\}$. Since

$$
r_{2}(x)=(x-1)(x+1)=x^{2}-1,
$$

we find for a weight of Jacobi type that

$$
w(x) r_{2}(x)=(1-x)^{\alpha}(1+x)^{\beta}\left(x^{2}-1\right)=-(1-x)^{\alpha+1}(1+x)^{\beta+1} .
$$

For notational convenience, we let $w_{j}$ denote the Gaussian quadrature weights with respect to the positive weight function $(1-x)^{\alpha+1}(1+x)^{\beta+1}$, while $\tilde{w}_{j}$ denotes the corresponding interior weights of the Gauss-Lobatto quadrature rule for the Jacobi weight function $w(x)=(1-x)^{\alpha}(1+x)^{\beta}$. Hence, both $w_{j}$ and $\tilde{w}_{j}$ are positive values. With this notation, the result of Lemma 3.1 should be modified so that

$$
\tilde{w}_{j}=-\frac{w_{j}}{r_{2}\left(x_{j}\right)}=\frac{w_{j}}{1-x_{j}^{2}}, \quad j=0, \ldots, n .
$$

It is easy to see that the interior nodes for Gauss-Lobatto integration with respect to $w(x)$ are the roots of $P_{n+1}^{(\alpha+1, \beta+1)}(x)$. These are precisely the roots of $P_{n+2}^{(\alpha, \beta) \prime}(x)$, or the extrema of $P_{n+2}^{(\alpha, \beta)}(x)$ in $(-1,1)$.

Theorem 3.2. Let $x_{j}$ be the roots of $P_{n+1}^{(\alpha+1, \beta+1)}(x)$ and denote by $\tilde{w}_{j}$ the corresponding interior weights of the Gauss-Lobatto quadrature rule for the Jacobi weight function $w(x)=(1-x)^{\alpha}(1+x)^{\beta}$. Then we may choose

$$
\tilde{\lambda}_{j}=C_{n}^{(\alpha+1, \beta+1)}(-1)^{j+1} \sqrt{\tilde{w}_{j}}, \quad j=0, \ldots, n,
$$

with $C_{n}^{(\alpha+1, \beta+1)}$ defined as in (2.25).

\footnotetext{
${ }^{1}$ We will consistently use the notation $\tilde{\lambda}$ and $\tilde{w}$ for barycentric weights and quadrature weights corresponding to the roots of an orthogonal polynomial, and $\hat{\lambda}$ and $\hat{w}$ for the added points.
} 
The corresponding barycentric weights for the points \pm 1 are

$$
\hat{\lambda}_{1}=C_{n}^{(\alpha+1, \beta+1)} \sqrt{(\beta+1) \hat{w}_{1}}
$$

and

$$
\hat{\lambda}_{2}=C_{n}^{(\alpha+1, \beta+1)}(-1)^{n} \sqrt{(\alpha+1) \hat{w}_{2}} .
$$

Proof. Let $\lambda_{j}^{x}$ be the barycentric weights corresponding to the point set $\left\{x_{j}\right\}_{j=0}^{n}$. From Corollary 2.3 we already know that

$$
\lambda_{j}^{x}=C_{n}^{(\alpha+1, \beta+1)}(-1)^{j} \sqrt{\left(1-x_{j}^{2}\right) w_{j}} .
$$

Combining this expression with (3.3) and (3.5) leads to

$$
\tilde{\lambda}_{j}=\frac{\lambda_{j}^{x}}{-\left(1-x_{j}^{2}\right)}=-C_{n}^{(\alpha+1, \beta+1)} \frac{(-1)^{j} \sqrt{\left(1-x_{j}^{2}\right) w_{j}}}{1-x_{j}^{2}}=C_{n}^{(\alpha+1, \beta+1)}(-1)^{j+1} \sqrt{\tilde{w}_{j}} .
$$

This shows (3.6).

It remains to determine the barycentric weights corresponding to the endpoints.

Let $k_{n+1}^{(\alpha+1, \beta+1)}$ denote the leading coefficients of the Jacobi polynomial $P_{n+1}^{(\alpha+1, \beta+1)}(x)$. It is known that (see [25, p. 59 and p. 63])

$$
k_{n+1}^{(\alpha+1, \beta+1)}=\frac{1}{2^{n+1}}\left(\begin{array}{c}
2 n+\alpha+\beta+4 \\
n+1
\end{array}\right), \quad P_{n+1}^{(\alpha+1, \beta+1)}(-1)=(-1)^{n+1}\left(\begin{array}{c}
n+\beta+2 \\
n+1
\end{array}\right) .
$$

We have from (3.4) that

$$
\begin{aligned}
\hat{\lambda}_{1} & =\frac{k_{n+1}^{(\alpha+1, \beta+1)}}{r_{2}^{\prime}(-1) P_{n+1}^{(\alpha+1, \beta+1)}(-1)} \\
& =-\frac{1}{2} k_{n+1}^{(\alpha+1, \beta+1)} \frac{1}{P_{n+1}^{(\alpha+1, \beta+1)}(-1)} \\
& =\frac{(-1)^{n}}{2^{n+2}} \frac{\Gamma(2 n+\alpha+\beta+5) \Gamma(\beta+2)}{\Gamma(n+\beta+3) \Gamma(n+\alpha+\beta+4)} .
\end{aligned}
$$

We have used $r_{2}(x)=x^{2}-1$, so that $r_{2}^{\prime}(-1)=-2$. From [10, Eqs. (3.10) and (3.11)] we know that

$$
\begin{aligned}
\hat{w}_{1} & =2^{\alpha+\beta+1} \frac{\Gamma(\alpha+2) \Gamma(\beta+1)}{\Gamma(\alpha+\beta+3)} \frac{\left(\begin{array}{c}
n+\alpha+2 \\
n+1
\end{array}\right)}{\left(\begin{array}{c}
n+\beta+2 \\
n+1
\end{array}\right)\left(\begin{array}{c}
n+\alpha+\beta+3 \\
n+1
\end{array}\right)} \\
& =2^{\alpha+\beta+1} \frac{\Gamma(\beta+1) \Gamma(n+\alpha+3) \Gamma(\beta+2)(n+1) !}{\Gamma(n+\beta+3) \Gamma(n+\alpha+\beta+4)}
\end{aligned}
$$

and

$$
\begin{aligned}
\hat{w}_{2} & =2^{\alpha+\beta+1} \frac{\Gamma(\beta+2) \Gamma(\alpha+1)}{\Gamma(\alpha+\beta+3)} \frac{\left(\begin{array}{c}
n+\beta+2 \\
n+1
\end{array}\right)}{\left(\begin{array}{c}
n+\alpha+2 \\
n+1
\end{array}\right)\left(\begin{array}{c}
n+\alpha+\beta+3 \\
n+1
\end{array}\right)} \\
& =2^{\alpha+\beta+1} \frac{\Gamma(\alpha+1) \Gamma(n+\beta+3) \Gamma(\alpha+2)(n+1) !}{\Gamma(n+\alpha+3) \Gamma(n+\alpha+\beta+4)} .
\end{aligned}
$$


Motivated by the form of (3.6), after some calculations, we find that

$$
\begin{aligned}
\left(C_{n}^{(\alpha+1, \beta+1)}\right)^{2} \hat{w}_{1} & =\frac{\Gamma(2 n+\alpha+\beta+5)^{2} \Gamma(\beta+1) \Gamma(\beta+2)}{2^{2 n+4} \Gamma(n+\beta+3)^{2} \Gamma(n+\alpha+\beta+4)^{2}} \\
& =\frac{1}{\beta+1}\left(\frac{\Gamma(2 n+\alpha+\beta+5) \Gamma(\beta+2)}{2^{n+2} \Gamma(n+\beta+3) \Gamma(n+\alpha+\beta+4)}\right)^{2} \\
& =\frac{\hat{\lambda}_{1}^{2}}{\beta+1} .
\end{aligned}
$$

Hence,

$$
\hat{\lambda}_{1}^{2}=\left(C_{n}^{(\alpha+1, \beta+1)}\right)^{2}(\beta+1) \hat{w}_{1} .
$$

Similarly, we can show that

$$
\hat{\lambda}_{2}^{2}=\left(C_{n}^{(\alpha+1, \beta+1)}\right)^{2}(\alpha+1) \hat{w}_{2} .
$$

Again noting that the barycentric weights have alternating signs, expressions (3.7) and (3.8) follow.

Note for completeness that in Theorem 3.2 we consider interpolation in a set of $n+3$ points in total. These points are

$$
\{-1,1\} \cup\left\{x_{j}\right\}_{j=0}^{n},
$$

where $x_{j}$ are the $n+1$ roots of $P_{n+1}^{(\alpha+1, \beta+1)}$. In our current notation, the weights of the corresponding Gauss-Jacobi-Lobatto quadrature rule, relative to the weight function $(1-x)^{\alpha}(1+x)^{\beta}$, are

$$
\left\{\hat{w}_{1}, \hat{w}_{2}\right\} \cup\left\{\tilde{w}_{j}\right\}_{j=0}^{n} .
$$

The result of Theorem 3.2 may be written more concisely as follows.

Corollary 3.3. Let $-1=x_{0}<x_{1}<\cdots<x_{n}=1$ be the roots of $\left(1-x^{2}\right) P_{n}^{(\alpha, \beta) \prime}(x)$ and let $\left\{w_{j}\right\}_{j=0}^{n}$ be the corresponding weights of the interpolatory quadrature rule associated with the weight function $(1-x)^{\alpha}(1+x)^{\beta}$. Then for $n \geq 1$, the barycentric weights for the interpolation points $\left\{x_{j}\right\}_{j=0}^{n}$ are

$$
\lambda_{j}=C_{n-2}^{(\alpha+1, \beta+1)}(-1)^{j} \sqrt{\delta_{j} w_{j}}, \quad \delta_{j}= \begin{cases}\beta+1, & j=0, \\ \alpha+1, & j=n, \\ 1, & \text { otherwise } .\end{cases}
$$

The simplified barycentric weights can be obtained directly by deleting the factor $C_{n-2}^{(\alpha+1, \beta+1)}$.

We are especially concerned with some special cases of the Gauss-Jacobi-Lobatto points. When $\alpha=\beta=-1 / 2$, this corresponds to the Gauss-Chebyshev-Lobatto points, which are also called Chebyshev points of the second kind or ClenshawCurtis points. The Gauss-Chebyshev-Lobatto quadrature rule is given by

$$
\int_{-1}^{1} \frac{f(x)}{\sqrt{1-x^{2}}} d x \simeq \frac{\pi}{n} \sum_{k=0}^{n} \prime \prime f\left(x_{k}\right)
$$

where the double prime denotes a sum whose first and last terms are halved and the Gauss-Chebyshev-Lobatto points $x_{k}$ are given explicitly in (2.6). The following corollary is an immediate consequence of Corollary 3.3 . 
Corollary 3.4. For Gauss-Chebyshev-Lobatto points, the simplified barycentric weights are given by

$$
\lambda_{j}^{\mathrm{CH} 2}=(-1)^{j} \delta_{j}, \quad \delta_{j}=\left\{\begin{array}{cc}
1 / 2, & j=0 \text { or } j=n, \\
1, & \text { otherwise. }
\end{array}\right.
$$

Thus, we have provided an alternative simple derivation of the barycentric weights for the Chebyshev points of the second kind.

Another important case of $\alpha=\beta=0$ corresponds to the Gauss-LegendreLobatto points. The Gauss-Legendre-Lobatto quadrature rule is defined by

$$
\int_{-1}^{1} f(x) d x \simeq \sum_{j=0}^{n} w_{j} f\left(x_{j}\right)
$$

where the Gauss-Legendre-Lobatto points $\left\{x_{j}\right\}_{j=0}^{n}$ are the zeros of $\left(1-x^{2}\right) P_{n}^{\prime}(x)$ and $P_{n}(x)$ is the Legendre polynomial of degree $n$. The following corollary gives the simplified barycentric weights for the Gauss-Legendre-Lobatto points.

Corollary 3.5. For Gauss-Legendre-Lobatto points, the simplified barycentric weights $\lambda_{j}^{\mathrm{GLL}}$ are given by

$$
\lambda_{j}^{\mathrm{GLL}}=(-1)^{j} \sqrt{w_{j}}, \quad j=0, \ldots, n,
$$

where $w_{j}$ are the Gauss-Legendre-Lobatto quadrature weights.

Proof. It follows readily from Corollary 3.3.

Below, we list the steps for computing the interpolant that interpolates $f(x)$ at the Gauss-Jacobi-Lobatto points, i.e., roots of $\left(1-x^{2}\right) P_{n}^{(\alpha, \beta) \prime}(x)$.

Algorithm 1. Computation of the Gauss-Jacobi-Lobatto interpolant:

(1) Compute the nodes and weights of the $(n-1)$-point Gauss-Jacobi quadrature with respect to the weight $(1-x)^{\alpha+1}(1+x)^{\beta+1}$ by the Hale-Townsend algorithm [14. The interpolation points are the $(n-1)$ nodes supplemented with two additional points \pm 1 .

(2) Calculate the interior Gauss-Jacobi-Lobatto quadrature weights by (3.5) and compute the two boundary quadrature weights by their explicit expressions.

(3) Evaluate the barycentric weights by the Corollary 3.3 .

(4) Compute the Gauss-Jacobi-Lobatto interpolant by its barycentric representation.

In step 2, the two boundary quadrature weights can be computed directly if $n$ is smaller than 100. When $n$ is larger than 100 , however, direct evaluation of both boundary quadrature weights would result in an overflow. This problem can be avoided by reformulating both boundary quadrature weights via logarithms. 
3.3. Gauss-Radau variant for Jacobi polynomials. The Gauss-Radau variant is one where we include only the left endpoint $x=-1$. In that case, we have

$$
r_{1}(x)=x+1 .
$$

Thus, the other quadrature points are the roots of $P_{n+1}^{(\alpha, \beta+1)}$. We have the following result.

Theorem 3.6. Let $\left\{x_{j}\right\}_{j=0}^{n}$ be the roots of $P_{n+1}^{(\alpha, \beta+1)}(x)$ and denote by $\tilde{w}_{j}$ the corresponding weights of the Gauss-Jacobi-Radau quadrature rule with respect to the Jacobi weight function $w(x)=(1-x)^{\alpha}(1+x)^{\beta}$. Then the barycentric weights corresponding to the interior nodes $x_{j}$ are given by

$$
\tilde{\lambda}_{j}=C_{n}^{(\alpha, \beta+1)}(-1)^{j} \sqrt{\left(1-x_{j}\right) \tilde{w}_{j}}, \quad j=0, \ldots, n .
$$

The barycentric weight corresponding to the point $x=-1$ is

$$
\hat{\lambda}_{1}=-C_{n}^{(\alpha, \beta+1)} \sqrt{2(\beta+1) \hat{w}_{1}} .
$$

Proof. Let $\lambda_{j}^{x}$ be the barycentric weights corresponding to the point set $\left\{x_{j}\right\}_{j=0}^{n}$. From (3.3) and Corollary 2.3, it follows that

$$
\tilde{\lambda}_{j}=\frac{\lambda_{j}^{x}}{1+x_{j}}=\frac{C_{n}^{(\alpha, \beta+1)}(-1)^{j} \sqrt{\left(1-x_{j}^{2}\right) w_{j}}}{1+x_{j}}=C_{n}^{(\alpha, \beta+1)}(-1)^{j} \sqrt{\left(1-x_{j}\right) \tilde{w}_{j}}
$$

This proves (3.12). For the barycentric weight corresponding to the point $x=-1$, applying (3.4) yields

$$
\begin{aligned}
\hat{\lambda}_{1} & =\frac{k_{n+1}^{(\alpha, \beta+1)}}{r_{1}^{\prime}(-1) P_{n+1}^{(\alpha, \beta+1)}(-1)} \\
& =\frac{(-1)^{n+1} \Gamma(2 n+\alpha+\beta+4) \Gamma(\beta+2)}{2^{n+1} \Gamma(n+\beta+3) \Gamma(n+\alpha+\beta+3)} .
\end{aligned}
$$

On the other hand, from [1] we have

$$
\begin{aligned}
\hat{w}_{1} & =\frac{2^{\alpha+\beta+1} \Gamma(\beta+1) \Gamma(n+\alpha+2)}{\left(\begin{array}{c}
n+\beta+2 \\
n+1
\end{array}\right) \Gamma(n+\alpha+\beta+3)} \\
& =\frac{2^{\alpha+\beta+1} \Gamma(\beta+1) \Gamma(\beta+2) \Gamma(n+\alpha+2) \Gamma(n+2)}{\Gamma(n+\beta+3) \Gamma(n+\alpha+\beta+3)}
\end{aligned}
$$

and, by some computations,

$$
\begin{aligned}
\left(C_{n}^{(\alpha, \beta+1)}\right)^{2} \hat{w}_{1} & =\frac{\Gamma(\beta+1) \Gamma(\beta+2) \Gamma(2 n+\alpha+\beta+4)^{2}}{2^{2 n+3} \Gamma(n+\alpha+\beta+3)^{2} \Gamma(n+\beta+3)^{2}} \\
& =\frac{1}{2(\beta+1)} \frac{\Gamma(\beta+2)^{2} \Gamma(2 n+\alpha+\beta+4)^{2}}{2^{2 n+2} \Gamma(n+\alpha+\beta+3)^{2} \Gamma(n+\beta+3)^{2}} \\
& =\frac{\hat{\lambda}_{1}^{2}}{2(\beta+1)} .
\end{aligned}
$$

Thus, we find

$$
\hat{\lambda}_{1}^{2}=2(\beta+1)\left(C_{n}^{(\alpha, \beta+1)}\right)^{2} \hat{w}_{1} .
$$

Since the barycentric weights have alternating signs, expression (3.13) follows. 
Note for completeness that in the last theorem we consider barycentric interpolation in a set of $n+2$ points in total. These points are

$$
\{-1\} \cup\left\{x_{j}\right\}_{j=0}^{n},
$$

where $x_{j}$ are the $n+1$ roots of $P_{n+1}^{(\alpha, \beta+1)}$. In our current notation, the corresponding Gauss-Radau quadrature weights are

$$
\left\{\hat{w}_{1}\right\} \cup\left\{\tilde{w}_{j}\right\}_{j=0}^{n} .
$$

The result of Theorem 3.6 may be written more concisely as follows.

Corollary 3.7. Let $-1=x_{0}<x_{1}<\cdots<x_{n}<1$ be the roots of $(1+x) P_{n}^{(\alpha, \beta+1)}(x)$ and let $w_{j}$ be the corresponding weights of the interpolatory quadrature rule with the weight function $(1-x)^{\alpha}(1+x)^{\beta}$. Then the simplified barycentric weights are

$$
\lambda_{j}=(-1)^{j} \sqrt{\left(1-x_{j}\right) \delta_{j} w_{j}}, \quad \delta_{j}=\left\{\begin{array}{cc}
\beta+1, & j=0 \\
1, & \text { otherwise. }
\end{array}\right.
$$

Similar results hold if one chooses to add the other endpoint $x=+1$ instead.

We remark that the steps for computing the Gauss-Jacobi-Radau interpolant are similar to the Lobatto case. We omit the details.

3.4. Gauss-Radau variant for Laguerre polynomials. Finally, we consider a Radau variant for Laguerre polynomials. We include the left endpoint $x=0$ of the half-infinite integration interval $[0, \infty)$ as a pre-assigned quadrature point and thus we have $r_{1}(x)=x$. The result is the following.

Theorem 3.8. Let $x_{j}$ be the roots of $L_{n+1}^{(\alpha+1)}(x)$ and denote by $\tilde{w}_{j}$ the corresponding weights of the Gauss-Laguerre-Radau quadrature rule with respect to the Laguerre weight function $w(x)=x^{\alpha} e^{-x}$. Then the barycentric weights corresponding to the interior nodes $x_{j}$ are given by

$$
\tilde{\lambda}_{j}=C_{n}^{(\alpha+1)}(-1)^{j} \sqrt{\tilde{w}_{j}}, \quad j=0, \ldots, n .
$$

The barycentric weight corresponding to the point $x=0$ is

$$
\hat{\lambda}_{1}=-C_{n}^{(\alpha+1)} \sqrt{(\alpha+1) \hat{w}_{1}},
$$

where $C_{n}^{(\alpha+1)}$ is defined as in (2.27).

Proof. Let $\lambda_{j}^{x}$ be the barycentric weights corresponding to the point set $\left\{x_{j}\right\}_{j=0}^{n}$. By virtue of (3.3) and Corollary 2.4 yields

$$
\tilde{\lambda}_{j}=\frac{\lambda_{j}^{x}}{x_{j}}=C_{n}^{(\alpha+1)}(-1)^{j} \sqrt{\frac{w_{j}}{x_{j}}}=C_{n}^{(\alpha+1)}(-1)^{j} \sqrt{\tilde{w}_{j}} .
$$

Let $k_{n+1}$ denote the leading coefficient of the Laguerre polynomial $L_{n+1}^{(\alpha+1)}(x)$. For the barycentric weight corresponds to the point $x=0$, using (3.4), we have that

$$
\hat{\lambda}_{1}=\frac{k_{n+1}}{L_{n+1}^{(\alpha+1)}(0)}=(-1)^{n+1} \frac{\Gamma(\alpha+2)}{\Gamma(n+\alpha+3)} .
$$

From [11, Eq. (6.5)] we have

$$
\hat{w}_{1}=\frac{\Gamma(\alpha+1)}{\left(\begin{array}{c}
n+\alpha+2 \\
n+1
\end{array}\right)}=\frac{\Gamma(\alpha+1) \Gamma(\alpha+2) \Gamma(n+2)}{\Gamma(n+\alpha+3)},
$$


and hence, by direct computation,

$$
\begin{aligned}
\left(C_{n}^{(\alpha+1)}\right)^{2} \hat{w}_{1} & =\frac{\Gamma(\alpha+1) \Gamma(\alpha+2)}{\Gamma(n+\alpha+3)^{2}} \\
& =\frac{1}{\alpha+1}\left(\frac{\Gamma(\alpha+2)}{\Gamma(n+\alpha+3)}\right)^{2} \\
& =\frac{1}{\alpha+1} \hat{\lambda}_{1}^{2} .
\end{aligned}
$$

Equivalently,

$$
\hat{\lambda}_{1}^{2}=(\alpha+1)\left(C_{n}^{(\alpha+1)}\right)^{2} \hat{w}_{1}
$$

Noting that barycentric weights have alternating signs, we obtain the result.

\section{Numerical EXAMPLES}

In this section we shall show several numerical examples to illustrate the performance of the barycentric interpolation formula. All computations were performed in Matlab in double precision arithmetic.

Example 4.1. We first consider the convergence of the barycentric Jacobi interpolation formula to the two smooth functions $f(x)=\frac{1}{1+25 x^{2}}$ and $f(x)=e^{-\frac{1}{x^{2}}}$.

The maximal pointwise error of the barycentric Jacobi formula

$$
\max _{-1 \leq x \leq 1}\left|f(x)-p_{n}(x)\right|
$$

is estimated by measuring at a large number of equispaced points in $[-1,1]$. The nodes and weights of the Gauss-Jacobi quadrature rule are computed with the Glaser-Liu-Rokhlin algorithm in $\mathcal{O}(n)$ operations. This computation can be performed in the Matlab package Chebfun with the command jacpts [15. Starting with Chebfun version 4.0, this routine returns the simplified barycentric weights as well, using formula (2.24) 2 . Future versions of this routine are likely to be based on the faster Hale-Townsend algorithm [14, but that will not change the asymptotic computational complexity of the experiment.

Figure 1 shows the convergence of the barycentric Jacobi formula with $\alpha=-\frac{1}{2}$ and $\beta=-\frac{1}{4}$. We can see that the barycentric Jacobi formula leads to stable computations. For large $\alpha$ and $\beta$, the Lebesgue constant for Jacobi points becomes very large, typically $\mathcal{O}\left(n^{\max \{\alpha, \beta\}+\frac{1}{2}}\right)$ [25, p. 338]. Hence, the barycentric Jacobi formula will be unstable. Figure 2 shows the convergence of the barycentric Jacobi formula for the same two functions with $\alpha=\beta=5$. We can see that the barycentric Jacobi formula is indeed unstable for large $n$, confirming the stability analysis of the barycentric formula by Higham in [17].

\footnotetext{
${ }^{2}$ The simplified barycentric weights returned by the command jacpts, lagpts, hermpts are normalized by setting their maximum value to one.
} 

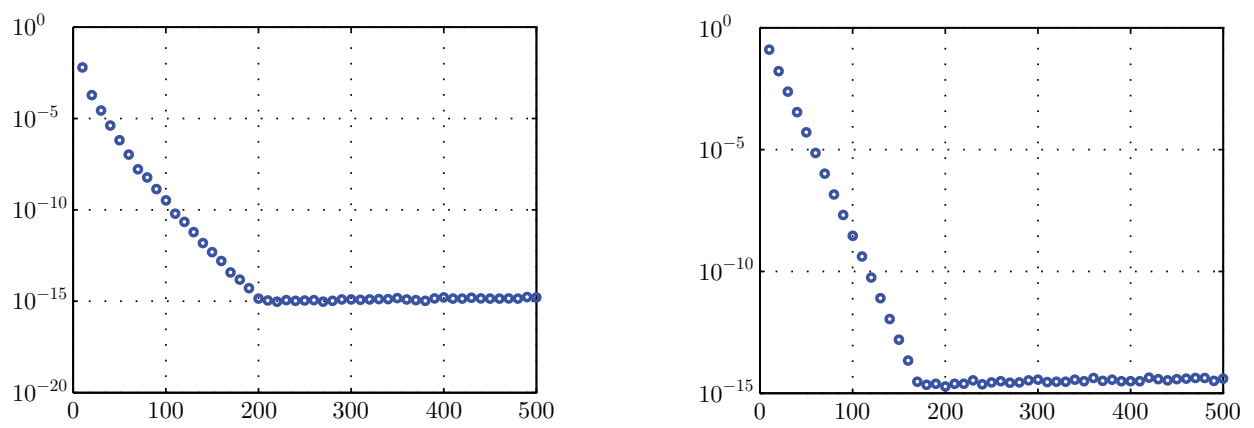

FiguRE 1. Convergence of the barycentric Jacobi formula to the function $f(x)=\frac{1}{1+25 x^{2}}$ (left) and $f(x)=e^{-\frac{1}{x^{2}}}$ (right). Here we choose $\alpha=-\frac{1}{2}, \beta=-\frac{1}{4}$ and $n$ ranges from 10 to 500 .
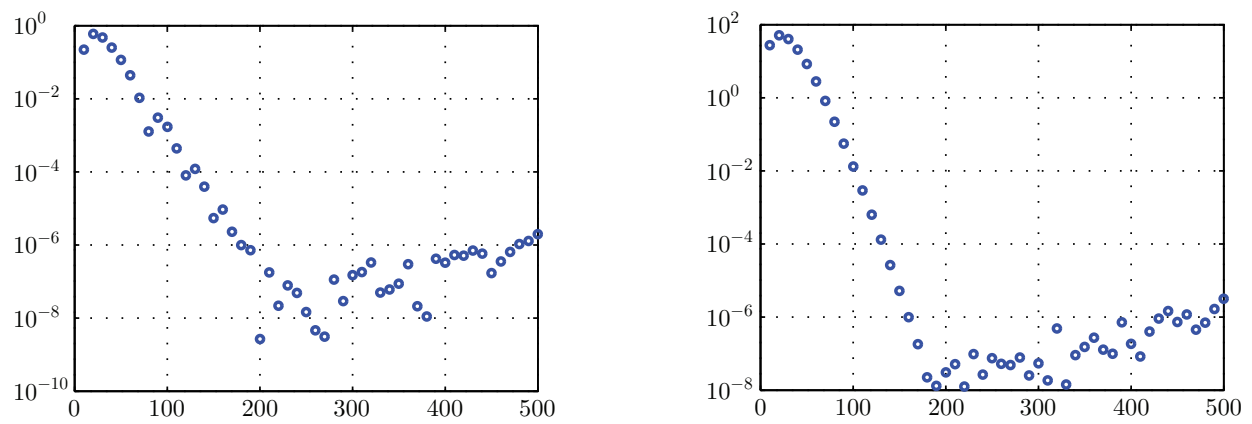

Figure 2. Convergence of the barycentric Jacobi formula to the two functions $f(x)=\frac{1}{1+25 x^{2}}$ (left) and $f(x)=e^{-\frac{1}{x^{2}}}$ (right). Here, we choose $\alpha=5, \beta=5$ and $n$ ranges from 10 to 500 .

Example 4.2. Next, we consider the application of the barycentric Jacobi interpolation formula to the function $J_{\frac{1}{3}}(x)$ on the interval $[0,1]$, where $J_{\frac{1}{3}}(x)$ is the Bessel function of the first kind of order $\frac{1}{3}$. Since the function $J_{\frac{1}{3}}(x)$ behaves like $x^{\frac{1}{3}}$ when $x \rightarrow 0$, we interpolate the function

$$
f(x)=\frac{J_{\frac{1}{3}}(x)}{x^{\frac{1}{3}}}, \quad x \in[0,1] .
$$

We apply the following norm to measure the error of the barycentric interpolation formula:

$$
\int_{0}^{1} x^{\frac{1}{3}}\left|f(x)-p_{n}(x)\right|^{2} d x .
$$

It is easy to see that this example corresponds to $\alpha=0$ and $\beta=\frac{1}{3}$. We have applied the barycentric Jacobi and Jacobi Lobatto formulae to approximate the function $f(x)$. The barycentric Jacobi weights are computed by (2.24) with $C_{n}^{(\alpha, \beta)}=1$. The barycentric Gauss-Jacobi-Lobatto formula is computed by using the Algorithm 1 and we have used the simplified barycentric Gauss-Jacobi-Lobatto weights in our implementation. Numerical results are illustrated in Figure 3. 

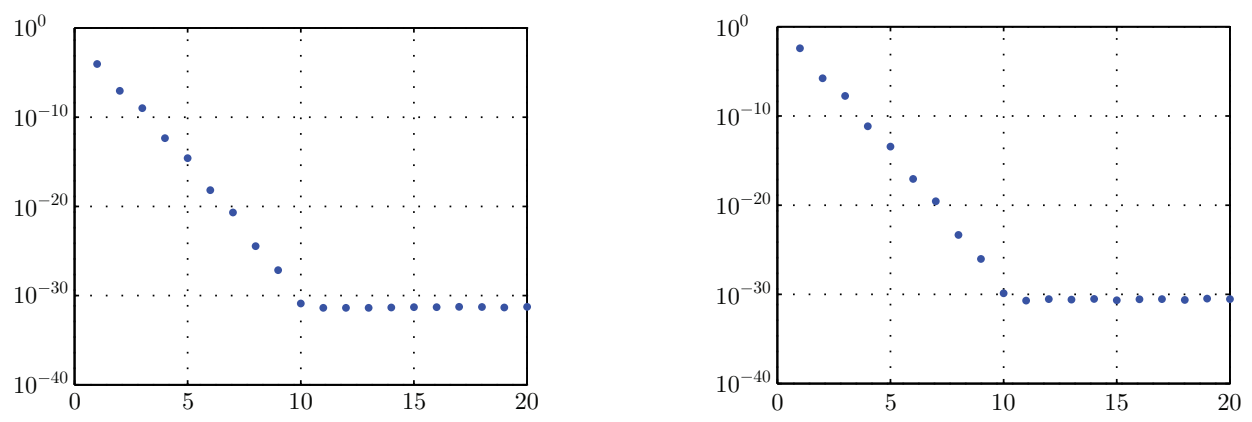

FiguRE 3. Convergence of the barycentric Jacobi (left) and Jacobi Lobatto formulas (right). Here $n$ ranges from 1 to 20 .
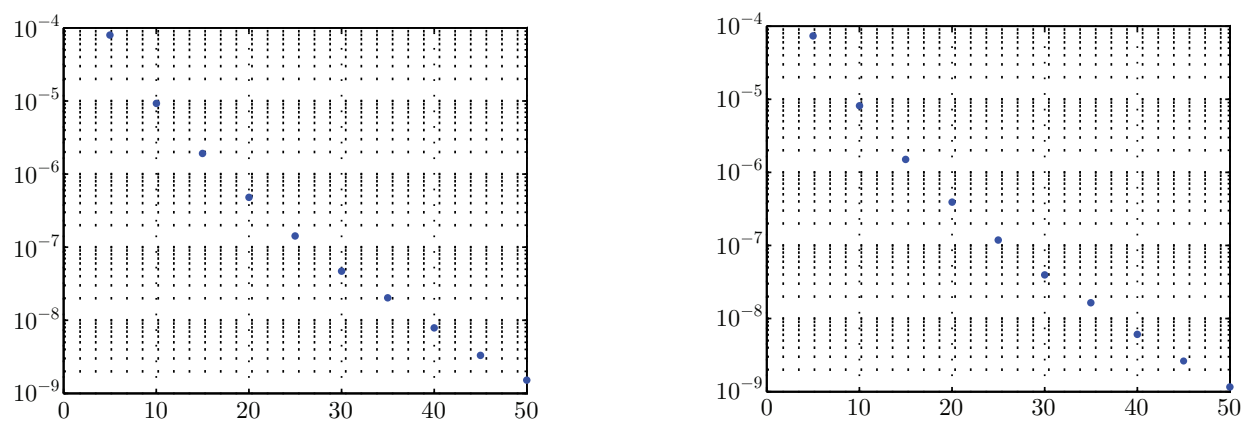

Figure 4. Convergence of the barycentric Laguerre (left) and Laguerre Radau (right) formulae to the function (4.1).

Example 4.3. Finally, we consider the application of the barycentric Laguerre interpolation formula to the function $\operatorname{Ai}\left(\left(\frac{3}{2}(x+1)\right)^{\frac{2}{3}}\right)$ on the interval $[0, \infty)$, where $\operatorname{Ai}(x)$ denotes the Airy function. Since this function behaves like $e^{-x}$ when $x \rightarrow \infty$, we interpolate the function

$$
f(x)=\operatorname{Ai}\left(\left(\frac{3}{2}(x+1)\right)^{\frac{2}{3}}\right) e^{x}, \quad x \in[0, \infty) .
$$

We apply the following norm to measure the error of the barycentric Laguerre interpolation formula:

$$
\int_{0}^{\infty} e^{-x}\left|f(x)-p_{n}(x)\right| d x
$$

For the barycentric Laguerre formula, the nodes and weights of Gauss-Laguerre quadrature are evaluated in Chebfun with the command lagpts, which also returns the simplified barycentric weights using the formula (2.26). For the barycentric Gauss-Laguerre-Radau formula, the nodes and weights are evaluated with the Golub-Welsch algorithm, which is based on computing eigenvalues and eigenvectors of a symmetric tridiagonal matrix whose elements are obtained from the three-term recurrence relation satisfied by the Laguerre polynomials [13]. The barycentric Gauss-Laguerre-Radau weights are computed by Theorem 3.8. Numerical results are shown in Figure 4. As we can see, both formulas are of approximately equal accuracy. 


\section{Conclusion}

We have investigated the fast computation of the interpolation polynomials based on the zeros or extrema of classical families of orthogonal polynomials. We have shown that the barycentric weights and the corresponding quadrature weights are intimately related to each other and that such relationships are a direct consequence of the existence of lowering operators for orthogonal polynomials. Note that the nodes and weights of the classical Gaussian quadrature formulas can be efficiently computed using the Glaser-Liu-Rokhlin algorithm for Laguerre and Hermite polynomials, and by the more efficient Hale-Townsend algorithm [14 for the Jacobi polynomials. The interpolation polynomials based on the zeros of these polynomials can thus be computed efficiently by using their barycentric representations.

The formulas for the barycentric weights for the Jacobi, Laguerre and Hermite polynomials were already described and implemented as part of the Chebfun package $[15,28$. We have extended the idea to the implementation of the barycentric interpolation in the extrema of these classical polynomials with some additional boundary points, e.g., Gauss-Radau and Gauss-Lobatto points. The link between the barycentric weights and the corresponding quadrature weights is established which allows the computation of the interpolants in Gauss-Radau and Gauss-Lobatto points in $\mathcal{O}(n)$ operations as well.

\section{ACKNOWLEDGEMENTS}

The authors would like to thank Alfredo Deaño and Lun Zhang for helpful discussions about the theory of lowering operators for orthogonal polynomials, and Jean-Paul Berrut and Nick Trefethen for their valuable comments on the history and recent developments of barycentric weights and their implementation in Chebfun.

\section{REFERENCES}

[1] Jean-Paul Berrut and Lloyd N. Trefethen, Barycentric Lagrange interpolation, SIAM Rev. 46 (2004), no. 3, 501-517 (electronic), DOI 10.1137/S0036144502417715. MR 2115059 (2005k:65018)

[2] I. Bogaert, B. Michiels, and J. Fostier, $\mathcal{O}(1)$ computation of Legendre polynomials and GaussLegendre nodes and weights for parallel computing, SIAM J. Sci. Comput. 34 (2012), no. 3, C83-C101, DOI 10.1137/110855442. MR2970283

[3] Yang Chen and Mourad E. H. Ismail, Ladder operators and differential equations for orthogonal polynomials, J. Phys. A 30 (1997), no. 22, 7817-7829, DOI 10.1088/0305-4470/30/22/020. MR1616931 (2000i:33011)

[4] E. W. Cheney, Introduction to approximation theory, McGraw-Hill Book Co., New York, 1966. MR0222517 (36 \#5568)

[5] Germund Dahlquist and Åke Björck, Numerical methods in scientific computing. Vol. I, Society for Industrial and Applied Mathematics (SIAM), Philadelphia, PA, 2008. MR2412832 (2009b:65003)

[6] Philip J. Davis, Interpolation and approximation, Dover Publications Inc., New York, 1975. Republication, with minor corrections, of the 1963 original, with a new preface and bibliography. MR0380189 (52 \#1089)

[7] Philip J. Davis and Philip Rabinowitz, Methods of numerical integration, 2nd ed., Computer Science and Applied Mathematics, Academic Press Inc., Orlando, FL, 1984. MR760629 (86d:65004) 
[8] A. Dutt, M. Gu, and V. Rokhlin, Fast algorithms for polynomial interpolation, integration, and differentiation, SIAM J. Numer. Anal. 33 (1996), no. 5, 1689-1711, DOI 10.1137/0733082. MR1411845 (97h:65015)

[9] Walter Gautschi, Numerical analysis, An introduction, Birkhäuser Boston, Inc., Boston, MA, 1997. MR 1454125(98d:65001)

[10] Walter Gautschi, High-order Gauss-Lobatto formulae, Mathematical journey through analysis, matrix theory and scientific computation (Kent, OH, 1999), Numer. Algorithms 25 (2000), no. 1-4, 213-222, DOI 10.1023/A:1016689830453. MR1827155(2002c:65043)

[11] Walter Gautschi, Gauss-Radau formulae for Jacobi and Laguerre weight functions, Math. Comput. Simulation 54 (2000), no. 4-5, 403-412, DOI 10.1016/S0378-4754(00)00179-8. 1999 International Symposium on Computational Sciences, to honor John R. Rice (West Lafayette, IN). MR.1808697 (2001k:65049)

[12] Andreas Glaser, Xiangtao Liu, and Vladimir Rokhlin, A fast algorithm for the calculation of the roots of special functions, SIAM J. Sci. Comput. 29 (2007), no. 4, 1420-1438, DOI 10.1137/06067016X. MR2341794(2009c:33056)

[13] Gene H. Golub and John H. Welsch, Calculation of Gauss quadrature rules, Math. Comp. 23 (1969), 221-230; addendum, ibid. 23 (1969), no. 106, loose microfiche suppl, A1-A10. MR.0245201 (39 \#6513)

[14] Nicholas Hale and Alex Townsend, Fast and accurate computation of Gauss-Legendre and Gauss-Jacobi quadrature nodes and weights, SIAM J. Sci. Comput. 35 (2013), no. 2, A652A674, DOI 10.1137/120889873. MR.3033086

[15] Nicholas Hale and Lloyd N. Trefethen, Chebfun and numerical quadrature, Sci. China Math. 55 (2012), no. 9, 1749-1760, DOI 10.1007/s11425-012-4474-z. MR2960859

[16] Peter Henrici, Essentials of numerical analysis with pocket calculator demonstrations, John Wiley \& Sons Inc., New York, 1982. MR655251 (83h:65002)

[17] Nicholas J. Higham, The numerical stability of barycentric Lagrange interpolation, IMA J. Numer. Anal. 24 (2004), no. 4, 547-556, DOI 10.1093/imanum/24.4.547. MR2094569 (2005e:65007)

[18] J. C. Mason and D. C. Handscomb, Chebyshev polynomials, Chapman \& Hall/CRC, Boca Raton, FL, 2003. MR.1937591 (2004h:33001)

[19] Arnold F. Nikiforov and Vasilii B. Uvarov, Special functions of mathematical physics, Birkhäuser Verlag, Basel, 1988. A unified introduction with applications; Translated from the Russian and with a preface by Ralph P. Boas; With a foreword by A. A. Samarskiu. MR.922041 (89h:33001)

[20] Rodrigo B. Platte, Lloyd N. Trefethen, and Arno B. J. Kuijlaars, Impossibility of fast stable approximation of analytic functions from equispaced samples, SIAM Rev. 53 (2011), no. 2, 308-318, DOI 10.1137/090774707. MR2806639 (2012e:41018)

[21] H. E. Salzer, Lagrangian interpolation at the Chebyshev points $x_{n, \nu} \equiv \cos (\nu \pi / n), \nu=0(1) n$; some unnoted advantages, Comput. J. 15 (1972), 156-159. MR0315865(47 \#4414)

[22] Jie Shen and Tao Tang, Spectral and high-order methods with applications, Mathematics Monograph Series, vol. 3, Science Press Beijing, Beijing, 2006. MR2723481 (2012b:65001)

[23] Eduard L. Stiefel, An introduction to numerical mathematics, Translated by Werner C. Rheinboldt and Cornelie J. Rheinboldt, Academic Press, New York, 1963. MR0181077(31 \#5306)

[24] Endre Süli and David F. Mayers, An introduction to numerical analysis, Cambridge University Press, Cambridge, 2003. MR2006500

[25] G. Szegő, Orthogonal Polynomials, Colloquium Publications 23, A, Providence, Rhode Island, 1939.

[26] Craig A. Tracy and Harold Widom, Fredholm determinants, differential equations and matrix models, Comm. Math. Phys. 163 (1994), no. 1, 33-72. MR1277933 (95e:82005)

[27] Lloyd N. Trefethen, Approximation theory and approximation practice, Society for Industrial and Applied Mathematics (SIAM), Philadelphia, PA, 2013. MR3012510

[28] L. N. Trefethen and others, Chebfun Version 4.0, The Chebfun Development Team, http://www.maths.ox.ac.uk/chebfun/, 2011.

[29] Haiyong Wang and Shuhuang Xiang, On the convergence rates of Legendre approximation, Math. Comp. 81 (2012), no. 278, 861-877, DOI 10.1090/S0025-5718-2011-02549-4. MR2869040(2012k:41021) 
[30] M. Webb, L. N. Trefethen and P. Gonnet, Stability of barycentric interpolation formulas for extrapolation, SIAM J. Sci. Comput., 34 (2012), A3009-A3015.

[31] Wilhelm Werner, Polynomial interpolation: Lagrange versus Newton, Math. Comp. 43 (1984), no. 167, 205-217, DOI 10.2307/2007406. MR744931(86g:65024)

School of Mathematics and Statistics, Huazhong University of Science and Technology, Wuhan 430074, People's Republic of China

E-mail address: haiyongwang@hust.edu.cn

Department of Computer Science, K.U.Leuven, Celestijnenlaan 200A, B-3001 Leuven, BELGIUM

E-mail address: daan.huybrechs@cs.kuleuven.be

Department of Computer Science, K.U.Leuven, Celestijnenlaan 200A, B-3001 Leuven, BELGiUM

E-mail address: stefan.vandewalle@cs.kuleuven.be 